\title{
1 Modelling the occurrence of heat waves in maximum 2 and minimum temperatures over Spain and 3 projections for the period 2031-60
}

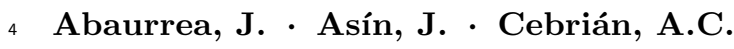 \\ 6 Received: date / Accepted: date
}

7 Abstract The occurrence of extreme heat events in maximum and minimum daily 8 temperatures is modelled using a non homogeneous common Poisson shock pro9 cess. It is applied to five Spanish locations, representative of the most common 10 climates over the Iberian Peninsula. The model is based on an excess over thresh11 old approach and distinguishes three types of extreme events: only in maximum

J. Abaurrea

University of Zaragoza

J. Asín

University of Zaragoza

A.C. Cebrián

University of Zaragoza

E-mail: acebrian@unizar.es 
jections for 2031-60 under scenarios RCP4.5, RCP6.0 and RCP8.5 are obtained and analysed using the trajectories from four earth system models which have successfully passed a preliminary control analysis. Different graphical tools and summary measures of the projected daily intensities are used to quantify the climate change on a local scale. A high increase in the occurrence of extreme heat events, mainly in July and August, is projected in all the locations, all types of event and in the three scenarios, although in 2051-60 the increase is higher under RCP8.5. However, relevant differences are found between the evolution in the different climates and the types of event, with a specially high increase in the simultaneous ones.

Keywords Extreme heat events $\cdot$ non homogeneous Poisson process $\cdot$ bivariate models $\cdot$ climate projections $\cdot$ climate change

\section{Introduction}

The analysis of heat waves is an increasingly important issue due to the serious impact of this phenomenon on ecosystems, the economy and human health; see for example Beniston et al (2007), Barriopedro et al (2011), Amengual et al (2014) and Tobías et al (2014). There is no standard definition of heat wave and many authors, such as Perkins and Alexander (2013) and Smith et al (2013), address the issue of analysing different measurements and definitions of this phenomenon. Traditionally, heat waves have been defined using daily maximum temperatures but there is an increasing number of definitions including information on both maximum and minimum daily temperatures; see for example Tryhorn and Risbey (2006), Keellings and Waylen (2014) or the definition by the U.S. National Weather Service. According to Hajat et al (2006), both temperatures should be considered to analyse the effect of heat waves on human health.

The global warming induced by the increasing concentration of greenhouse gases in the atmosphere during the $20^{\text {th }}$ century, and especially during its last decades, will probably continue. Many works, such as Meehl et al (2005), Tryhorn 
49 and Risbey (2006) and Lemonsu et al (2014), suggest that heat waves will become

50 more frequent. In this context, an important issue for preventing global warming

51 impacts is the characterization and future projection, on a local scale, of heat

52 waves including information on both maximum and minimum daily temperatures.

Temperature projections at a daily and local scale are often required, see Wang et al (2012), Casanueva et al (2013) and Lau and Nath (2014), who emphasise the interest of using a fine spatial resolution to investigate regional phenomena. Nowadays, Earth System Models (ESMs) are the best tool for obtaining future projections of atmospheric variables on a monthly or seasonal scale over broad areas. However, they are unable to provide reliable temperature trajectories on a daily and local scale, and cannot be directly used to project the extreme temperature behaviour of local daily series, see Yue et al (2016), Brands et al (2013), Cattiaux et al (2013), and Keellings and Waylen (2015) who find that AR5 models are not able to reproduce extremes over the $90^{t h}$ percentile. Regional Circulation Models (RCMs) neither guarantee an adequate reproduction of extreme temperature events. For example, Vautard et al (2013), using the RCM projections driven by ERA-Interim, find an overestimation of summer temperature extremes in Mediterranean regions and an underestimation over Scandinavia. They also conclude that the increase of the RCM resolution does not generally improve this deficiency. Grotjahn et al (2016) conclude that dynamic methods overestimate the frequency of heat waves and underestimate that of cold events.

In this context, the use of statistical models to obtain heat wave projections is essential for many applications which require daily projections at a local spatial scale, such as health studies linked to heat extremes in big cities and other climate change impact studies. Another advantage of the statistical models is that they are able to deal with non stationary situations, be it using non constant thresholds, Kyselý et al (2010), or parameters depending on time or other covariates, see Cheng et al (2014), García-Cueto et al (2014) and Abaurrea et al (2015b). 
In this work, a bivariate point process, the common Poisson shock process, is used to model the occurrence of extreme heat events (EHE) in maximum and minimum daily temperatures. This model improves the univariate approaches, such as those suggested by Abaurrea et al (2007), Furrer et al (2010) or Kyselý et al (2010), since it takes into account the dependence between the occurrence of extreme events in both temperatures. The model can be easily generalised to a non stationary framework by making its parameters be a function of time-varying covariates. Here, only temperature related covariates are considered but other type of variables could also be used. An advantage of this model is that it can be easily estimated using the R package NHPoisson, see Cebrián et al (2015).

The model can be used to obtain local projections of the occurrence rate of EHEs under climate change conditions. These conditions are represented by covariates obtained from the future temperature trajectories generated by ESMs, appropriately downscaled to fit the climate characteristics of the considered location. Summary measures of these projected daily intensities allow us to quantify the local climate change.

The methodology is summarised in Section 2. Section 3 describes the data: the temperature series from five Spanish locations and four ESM daily trajectories. Section 4 shows and compares the fitted models in these locations. In Section 5, projections under scenarios RCP4.5, RCP6.0 and RCP8.5 for the period 2031-60 are obtained and analysed. Section 6 summarises the most relevant conclusions.

\section{Methodology}

\subsection{Modelling the occurrence of extreme heat events}

Common Poisson shock process The modelling of extreme events in environmental sciences is often based on the excess over threshold (EOT) approach, where an extreme event is defined as a run of observations whose values exceed a reference threshold; see Coles (2001). There is a point process characterization of extreme 
value models which states that, under mild conditions and if the threshold is extreme enough, the occurrence of the extreme events follows a Poisson process. Since a heat wave may provoke extreme values both in maximum and minimum daily temperatures, a bivariate approach will improve the univariate models usually applied to characterize the ocurrence of EHEs. In particular, a bivariate point process with dependent marginal processes is a reasonable framework to jointly model the occurrence of EHEs. In this work, a common Poisson shock process (CPSP) is considered; see Abaurrea et al (2015b) for a full justification of this model. One of the advantages of this approach is that it can be easily adapted to non stationarity.

A bivariate CPSP assumes that there is an underlying Poisson process (PP) of shocks $N_{0}$ that can yield two different types of events. The counting processes of each type of event are the marginal processes $N_{1}$ and $N_{2}$. The CPSP assumes that dependence occurs by the simultaneity of the events, so that it can be decomposed into three independent indicator PPs $N_{(1)}, N_{(2)}$ and $N_{(12)}$, which include the events occurring only in process $N_{1}$, only in $N_{2}$, and those occurring simultaneously in both of them. Their intensities are denoted $\lambda_{(1)}, \lambda_{(2)}$ and $\lambda_{(12)}$, respectively, so that the intensities of the marginal processes $N_{1}=N_{(1)}+N_{(12)}$ and $N_{2}=$ $N_{(2)}+N_{(12)}$ are $\lambda_{1}=\lambda_{(1)}+\lambda_{(12)}$ and $\lambda_{2}=\lambda_{(2)}+\lambda_{(12)}$.

The CPSP can be generalised to the nonhomogeneous case, by allowing the indicator intensities to be a function of a vector of time-varying predictors $\mathbf{x}(t)$ and using a logarithmic link, $\lambda(t \mid \mathbf{x}(t))=\exp \left(\beta^{\prime} \mathbf{x}(t)\right)$. The predictors also help to model the dependence induced by the systematic part of the three intensities.

The estimation of this model reduces to the estimation of three independent nonhomogeneous PPs, which can be carried out by maximum likelihood, and the covariate selection by a forward approach based on likelihood ratio tests. A detailed example of the estimation of a nonhomogenous PP can be found in Abaurrea et al (2007) and it can be easily implemented using the R package, NHPoisson, previously mentioned. 
Definition of extreme heat events The use of the CPSP for modelling EHEs in maximum and minimum daily temperature series ( $T x_{t}$ and $T n_{t}$ herein) requires some previous operational definitions. In particular, the three indicator processes and the types of extreme events whose occurrence is modelled in each process have to be defined: $N_{(1)}$ is the process which includes the EHEs only in $T x_{t}, N_{(2)}$ includes the EHEs only in $T n_{t}$, and $N_{(12)}$ those occurring simultaneously in both temperatures. Following the EOT definition of extreme event, an EHE only in $T x_{t}$ is a run of consecutive days where $T x_{t}$ exceeds $U_{x}$ but $T n_{t}$ does not exceed $U_{n}$, being $U_{x}$ and $U_{n}$ the extreme thresholds of the corresponding temperature series. An EHE only in $T n_{t}$ is defined analogously, and a simultaneous EHE is a run of observations with $T x_{t}$ and $T n_{t}$ exceeding $U_{x}$ and $U_{n}$, respectively.

Predictors Since the final objective of the model is to obtain future projections of the occurrence of EHEs, only variables with reliable future projections should be considered as potential predictors. Three types of variables are used here.

- Seasonal terms: Given that temperature series show a seasonal behaviour, seasonal terms have to be included in the model. In this case, they are defined as the part of the annual harmonic signals corresponding to the period of the year under consideration.

- Short moving averages of temperature: The moving average of $T x_{t}$ and $T n_{t}$ in 15 or 31 day intervals around $t$, denoted by $T x_{m 15}, T n_{m 15}, T x_{m 31}$ and $T n_{m 31}$, and their corresponding polynomial terms are considered. The reason to use these signals is that the projections provided by ESMs of the temperature series on an aggregated time scale of 15 or more days are reliable, while the projections of daily temperatures are not.

- Interaction terms: Interaction terms between the harmonic and the temperature predictors.

Validation analysis. The assumptions to be checked in a CPSP model are that the three indicator processes are non homogeneous PPs mutually independent. 
The first assumption is checked using the Kolmogorov-Smirnov (KS) test for the distribution of the residuals, and the Pearson test for serial correlation. The independence assumption is checked with the bootstrap test developed by Abaurrea et al (2015a). The details of the validation techniques can be found in Abaurrea et al (2015b).

\subsection{Projection of the extreme events}

Once a suitable model is fitted, the projection of the occurrence of EHEs is obtained using as input the covariates built from the future temperature trajectories provided by the ESMs. It is noteworthy that the ESM trajectories have to be properly downscaled to fit the site climate characteristics, before using them as input. In effect, statistical downscaling procedures bridge the gap between the ESM output, which are averages in gridcells with areas larger than $1^{o} \times 1^{o}$, and the information at a local scale required by the model, see Gutiérrez et al (2013). In addition, a validation analysis of the quality of the downscaled ESM trajectories should be carried out before using them for projecting.

Validating a trajectory. Two aspects are considered in the validation analysis. The first is that the downscaled ESM trajectory in the historical scenario reproduces satisfactorily the distribution of the observed temperatures, in particular, its tail distribution. Three tools are suggested to check this assumption: two exploratory graphs, see Section 5.1.1, and the test developed by Rosenbaum (2005), which checks the equality of two multivariate distributions. This requirement is not fulfilled by the temperature variables on a daily scale, as previously mentioned.

The second aspect is a control to avoid extrapolation. In a statistical model, the values of the covariates used to obtain predictions, in this case the future downscaled ESM trajectories, should not extrapolate the range of values used to fit the model. In particular, the reason why decadal temperature trends have not been considered as potential covariates, is that most of the values of their 
future projections lead to extrapolation problems. That is also the reason why only medium-term projections can be obtained using short moving average temperature variables.

\section{Data}

\subsection{Observed data}

The daily maximum and minimum temperature series, measured in ${ }^{\circ} C$, of five Spanish locations (Zaragoza, Barcelona, Badajoz, Albacete and Burgos) are analysed in this work. These series have been provided by the Spanish meteorological agency, AEMET. Their geographical position and Köppen ${ }^{1}$ climate classification are shown in Figure 1. Three of the series are located in the northern half of Spain: Burgos with a Cfb climate, Barcelona sited on the Mediterranean coast with a Csa climate and Zaragoza, in the Ebro valley, with a transition climate between the previous two, Bsk. Albacete and Badajoz are located in the southern half, in the Mediterranean and Atlantic slopes, with Bsk and Csa climates, respectively. These

1 http://es.climate-dat.org/location/3316

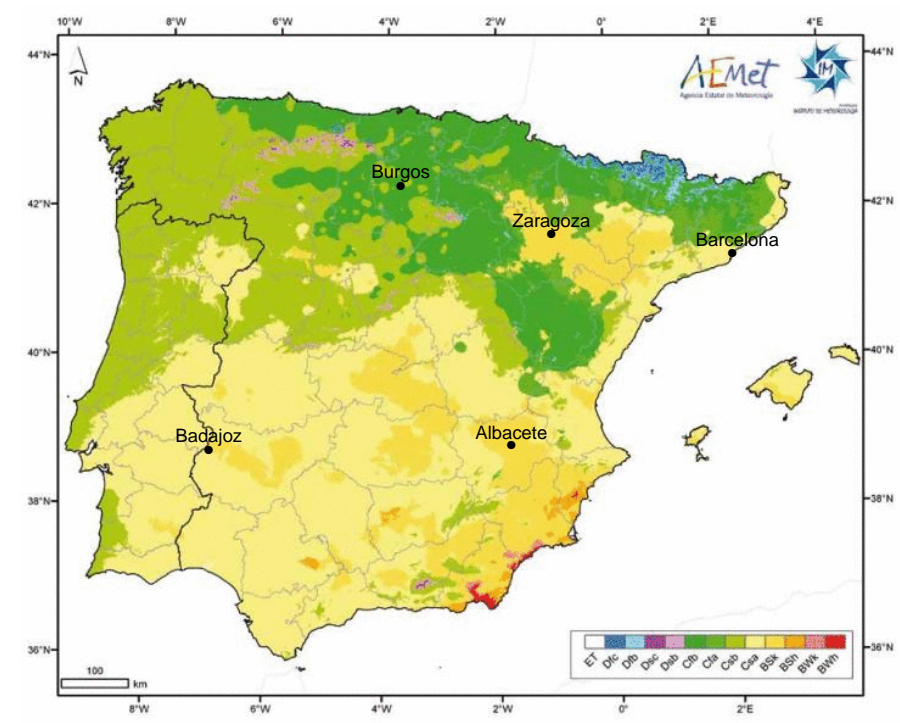

Fig. 1 Köppen classification and localization of the analysed series. Map from AEMET (2011). 
locations represent the most common climates in the Iberian Peninsula. It was no possible to analyse other climates since series of the required length and quality were not available.

In the Iberian peninsula summer runs from June to September, and an EHE has never been observed before May or after September. Consequently, the analysis of the occurrence of EHEs can be restricted to these months (MJJAS). The thresholds $U_{x}$ and $U_{n}$ used to characterize the EHEs in $T x_{t}$ and $T n_{t}$ are usually defined as percentiles of the observed series. The most common value is the $90^{\text {th }}$ percentile, see for example Tryhorn and Risbey (2006), but values between the $90^{\text {th }}$ and $99^{\text {th }}$ percentiles are also frequently used, see Hajat et al (2006). Since only Spanish series are considered in this work, and AEMET (2011) defines heat waves using as threshold the $95^{\text {th }}$ percentile of the daily temperature series from July to August in the reference period 1971-2000, that percentile is used to define $U_{x}$ and $U_{n}$.

Some characteristics of the $T x_{t}$ and $T n_{t}$ series are summarised in the first rows of Table 1: the altitude of the station, the record periods of $T x_{t}$ and $T n_{t}$ and their means in June, July, August and in the period MJJAS. The thresholds $U_{x}$ and $U_{n}$ are shown in the bottom part of Table 1, together with the observed number of EHEs in each indicator process.

\subsection{ESM Data}

Four CMIP5 climate models are used in this work, MPI-ESM-LR (MPI in short), CanESM2 (CE2), IPSL-CM5A-MR (IPSL) y MRI-CGCM3 (MRI). They are chosen for the quality of its representation of the summer climate patterns in the Atlantic area close to the Iberian Peninsula, among the CMIP5 models evaluated by Sánchez de Cos et al (2016).

Representative Concentration Pathways (RCPs) are greenhouse gas concentration trajectories which are consistent with a wide range of possible changes in future anthropogenic greenhouse gas emissions. In this work, three scenarios are 


\begin{tabular}{|l|lllll|}
\hline Series & Zaragoza & Barcelona & Badajoz & Albacete & Burgos \\
\hline Altitude (m. a.s.l.) & 263 & 412 & 185 & 702 & 891 \\
Record period & $1951-2005$ & $1951-2005$ & $1955-2005$ & $1961-2005$ & $1971-2008$ \\
$\overline{T x_{t}}$ MJJAS & 28.1 & 24.7 & 30.8 & 28.5 & 23.5 \\
$\overline{T x_{t}} \mathrm{Jn}$ & 27.7 & 24.1 & 30.3 & 27.9 & 22.0 \\
$\overline{T x_{t}} \mathrm{Jl}$ & 31.5 & 27.8 & 34.3 & 32.5 & 26.4 \\
$\overline{T x_{t}} \mathrm{Au}$ & 31.0 & 27.6 & 34.0 & 31.9 & 26.7 \\
$\overline{T n_{t}} \mathrm{MJJAS}$ & 15.1 & 16.3 & 14.9 & 13.3 & 9.1 \\
$\overline{T n_{t}} \mathrm{Jn}$ & 14.8 & 15.3 & 14.7 & 12.7 & 8.5 \\
$\overline{T n_{t}} \mathrm{Jl}$ & 17.6 & 18.6 & 17.0 & 16.0 & 11.0 \\
$\overline{T n_{t}} \mathrm{Au}$ & 17.8 & 18.7 & 16.7 & 16.1 & 11.1 \\
\hline$U_{x}$ & 37.0 & 31.8 & 39.6 & 37.0 & 33.2 \\
$U_{n}$ & 21.2 & 22.0 & 20.6 & 19.4 & 14.8 \\
\# EHE $N_{(1)}$ & 120 & 97 & 93 & 89 & 80 \\
$\#$ EHE $N_{(2)}$ & 92 & 114 & 124 & 117 & 89 \\
$\#$ EHE $N_{(12)}$ & 58 & 82 & 51 & 38 & 22 \\
\hline
\end{tabular}

Table 1 Summary values of $T x_{t}$ and $T n_{t}$ series (in ${ }^{\circ} C$ ), thresholds $U_{x}$ and $U_{n}$ used to define EHEs, and number of EHEs in each indicator process.

considered: RCP4.5 where emissions peak around 2040 and then decline, RCP6.0 where emissions peak around 2080 and then decline, and RCP8.5 where emissions continue to rise throughout the 21 st century. These scenarios are the most commonly used in climate change works, see Lau and Nath (2014) and Pereira et al (2017) for example, and they cover a range of different future scenarios from less to more pessimistic situations.

AEMET provides in its webpage ${ }^{2}$, the downscaled temperature series from more than 20 ESMs for different Spanish locations under scenarios RCP4.5 and RCP8.5 and in two of the ESMs also under RCP6.0. They are downscaled using a statistical procedure based on the regression method SDSM, see Wilby and Dawson (2013). In this work, the downscaled daily $T x$ and $T n$ trajectories of the previously described locations, Albacete, Badajoz, Barcelona, Burgos and Zaragoza, are needed. All of them, except Zaragoza, can be downloaded from the previous webpage. In that case, Leciñena series, around $35 \mathrm{~km}$ from Zaragoza, has been used after transforming it by correcting the mean level and the variability biases. Only

\footnotetext{
2 http://www.aemet.es/es/serviciosclimaticos/cambio_climat/datos_diarios
} 


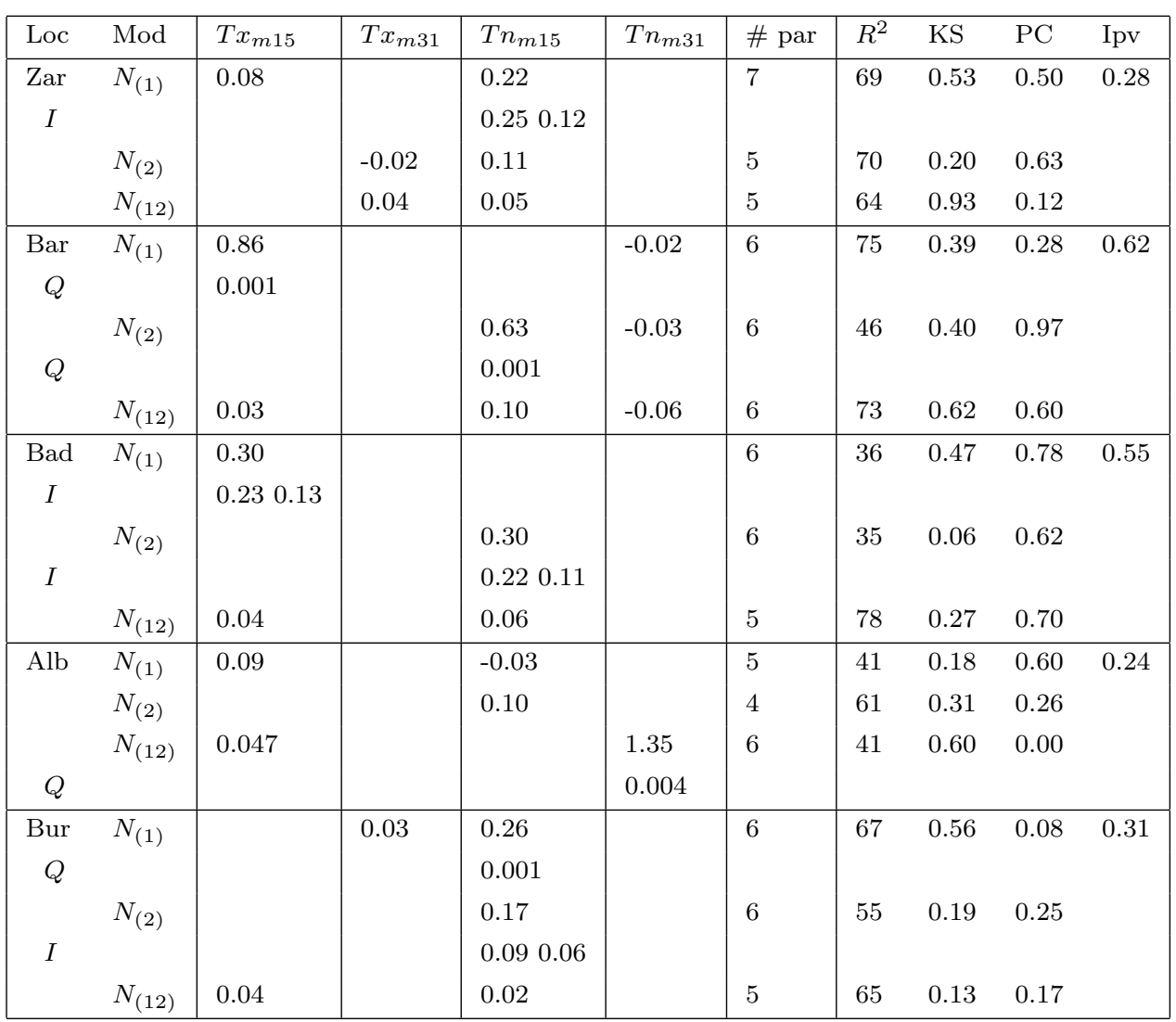

Table 2 Coefficients of the temperature covariates; interaction terms between the corresponding covariate and the harmonic, and quadratic terms are labeled $I$ and $Q$, respectively. Last columns: \# par, the number of model parameters, $R^{2}$ (in \%), and p-values of the KS test, the Pearson correlation test and the independence test.

two ESMs, IPSL and MRI, have projections for the scenario RCP6.0, so that only two trajectories are available in that case.

\section{Fitted Models}

A detailed example of the modelling process of a CPSP can be found in Abaurrea et al (2015b). The final models obtained following that approach are summarised in Table 2, where the coefficients of the significant temperature covariates are shown in the first columns. The rows labeled as $I$ correspond to the interaction terms between the corresponding covariate and the harmonic, and those labeled 
as $Q$ to the quadratic term of the temperature variables. The fitted models are quite simple, with between 4 and 7 parameters. The linear predictors of the three indicator processes $N_{(1)}, N_{(2)}$ and $N_{(12)}$ include, in all the locations, an intercept and one harmonic term. Only four, out of 15 fitted models, include a significant interaction term, and another four include a quadratic temperature term. As expected, the covariates based on 15-day moving averages are usually preferred over the 31-day averages.

At least one covariate related to $T x_{t}$ and another to $T n_{t}$ are significant in the $N_{(1)}$ models, except in Badajoz whose model only includes $T x_{m 15}$ and its interaction. The $T x_{t}$ terms have an increasing effect in all the locations, since even the quadratic effect in Barcelona is positive in the observed temperature range. High values of $T n_{t}$ (greater than $12^{\circ} \mathrm{C}$ in Burgos due to the quadratic term) lead to a reduction of the events in $N_{(1)}$, except in Zaragoza where the harmonic term gives a positive slope from the $10^{t h}$ July. This reduction can be explained by the fact that high $T n_{t}$ temperatures lead to an increase in the simultaneous events.

All the $N_{(2)}$ models include at least one $T n_{t}$ term, but only Zaragoza requires a covariate related to $T x_{t}$. The effect of $T n_{t}$ in all the locations increases the intensity in the observed temperature range, even the harmonic term in Badajoz and Burgos and the quadratic effect in Barcelona.

At least one covariate related to $T x_{t}$ and another to $T n_{t}$ are significant in the $N_{(12)}$ models. All the $T x_{t}$ terms have a positive linear trend while the effect of the $T n_{t}$ terms is also positive but not always linear.

The main results of the validation analysis are summarised in the last columns of Table 2: $R^{2}$ (the square correlation coefficient between the empirical and the fitted intensities), and the p-values of the KS, Pearson and the independence test, (see Section 2.1). All the models pass the validation analysis, and $R^{2}$ varies from 35 to $78 \%$. This coefficient is greater than 50 in $67 \%$ of the models. The empirical and fitted intensities, accumulated in periods of 5 months, are graphically compared 

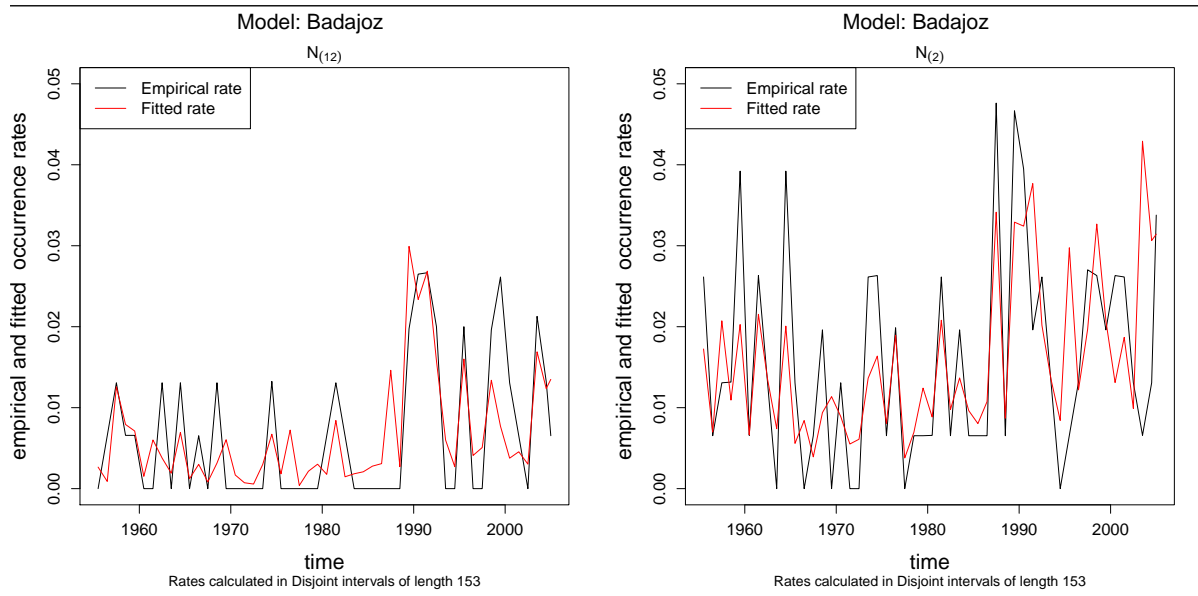

Fig. 2 Empirical and fitted intensities in Badajoz (models with the best and the worst fit).

with satisfactory results. As an example, the plots for the models with the best and the worst fit, Badajoz $N_{(12)}$ and $N_{(2)}$ respectively, are shown in Figure 2.

Figure 3 shows the LOWESS (with a 75 month window) of the three fitted intensities; for a better comparison the same y-scale is used in the three plots. A clear increase is observed from around the 90s in all the locations and types of event. Burgos shows one of the highest intensities in the tree types of event, while Zaragoza and Albacete are among the lowest. The high intensity of the simultaneous events in Barcelona is noteworthy. The greatest spatial variability is observed in $N_{(2)}$, with intensities in Burgos and Badajoz which are around four times the values in Zaragoza. The intensities of the three indicator processes show different levels. In all the locations, the highest intensities correspond to $N_{(2)}$, the medium ones to $N_{(1)}$ and the lowest to $N_{(12)}$, except for Zaragoza where the order of $N_{(2)}$ and $N_{(1)}$ is reversed.

\section{ESM projections}

In this section we obtain the projections under scenarios RCP4.5, RCP6.0 and RCP8.5 for the period 2031-60 using the ESM trajectories described in Section 3.2 and the fitted models discussed in Section 4. 

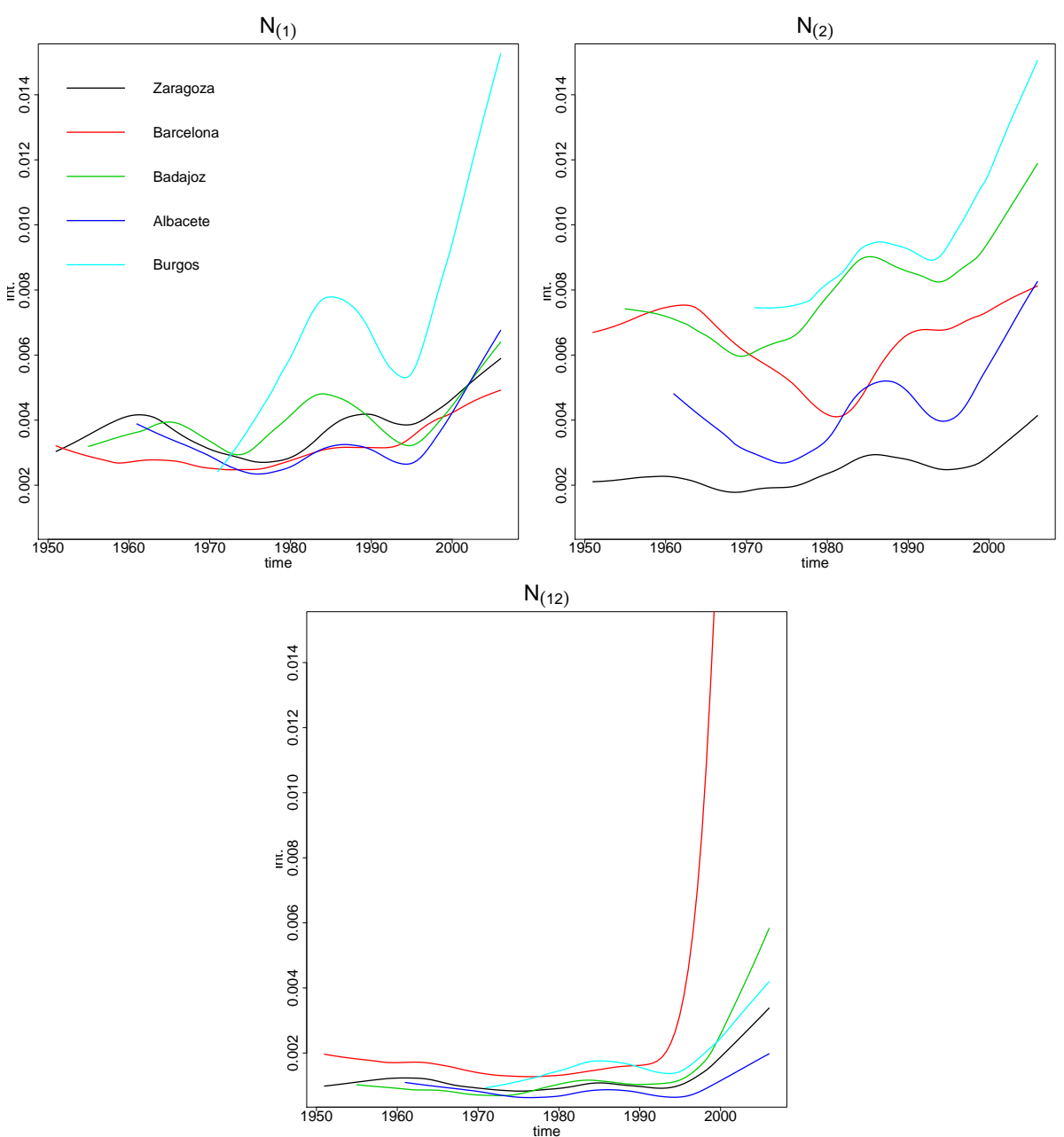

Fig. 3 Smoothed fitted intensities of the indicator processes. The y-scale in the plot for $N_{(12)}$ has been truncated from 0.05 (the maximum intensity in Barcelona) to 0.014.

5.1 Validating the trajectories

\subsubsection{Checking the ESM performance under the current climate conditions}

To check the performance of an ESM trajectory under the current climate conditions, the intensities fitted with the observed covariates are compared with those fitted with the corresponding downscaled historical trajectory. Since high intensities are of main interest, the comparison focuses on the high tails of the dis- 

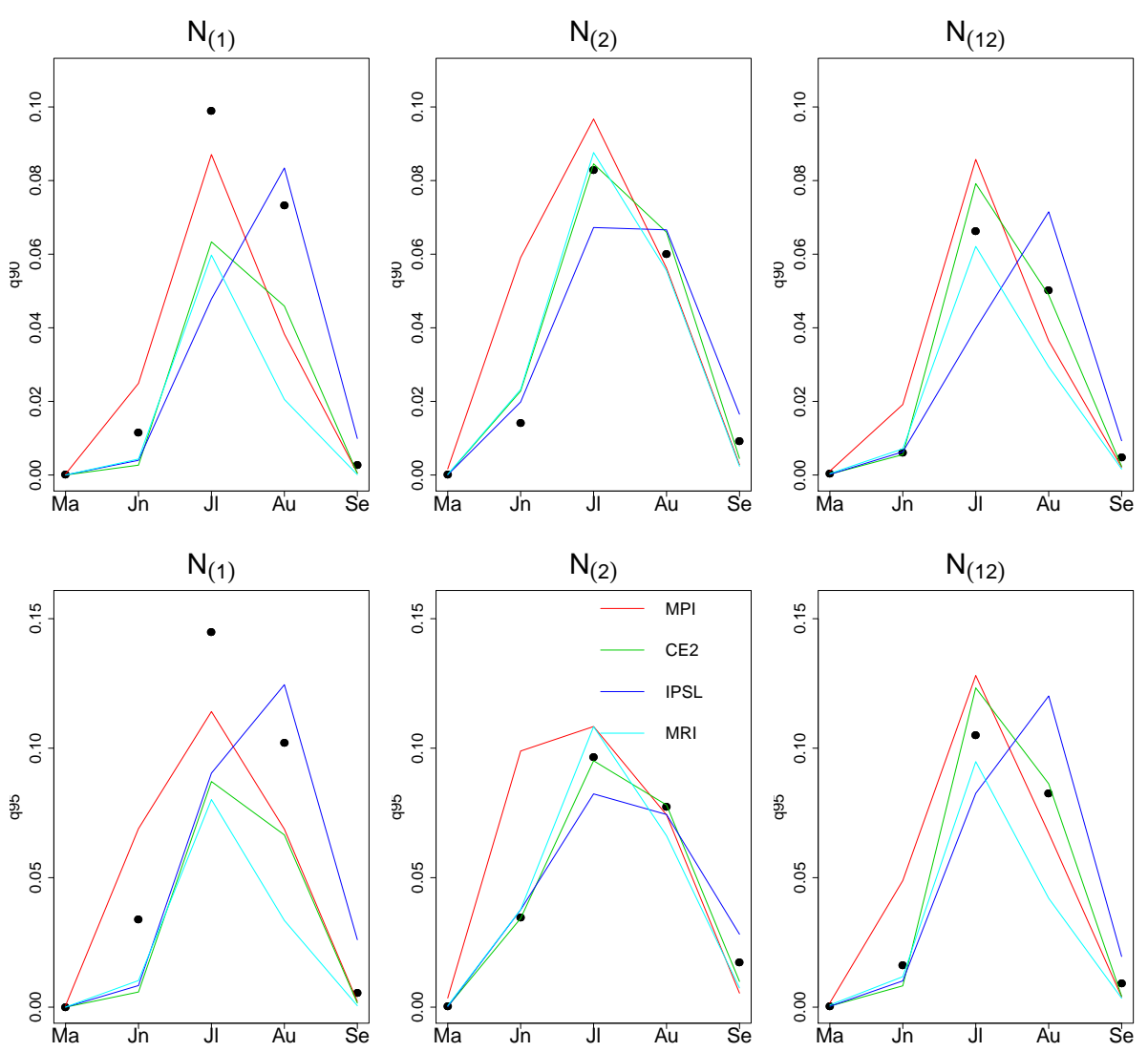

Fig. 4 Comparison of the observed (black points) and ESM percentiles for the historical scenario (lines), $90^{t h}$ percentile (top row) and $95^{\text {th }}$ percentile (bottom row), Barcelona.

tributions, using two plots and a test. Given the seasonal character of the EHE occurrence, this analysis is carried out separately for each month.

The first plot compares the percentiles of the intensities fitted with the observed covariates (observed percentiles herein) with those obtained from the available downscaled ESM trajectories (ESM percentiles). The plots for the $90^{\text {th }}$ and $95^{\text {th }}$ percentiles ( $q 90$ and $q 95$ ) of the indicator models in Barcelona, shown as an example in Figure 4, are satisfactory.

The boxplots of the observed and the ESM $95^{\text {th }}$ percentiles, by month, are used to check the inter-annual variability of the highest intensities. Each boxplot is based on a sample of 30 percentiles, one for each year during 1971-2000. The plots 
for Barcelona, Figure 5, show that the ESM historical scenarios are compatible with the observed ones. The dispersion of CE2 in May and June is much higher than the other ESMs, in the three types of events. The same applies to MPI in September.

Finally, the Rosenbaum test is applied to compare the observed and the ESM bivariate distribution of the $90^{t h}$ and the $95^{\text {th }}$ percentiles. A comparison for each available trajectory and month is applied, using the same samples as in the previous boxplots. The results show that only $3 \%$ of the 300 trajectories ( 5 months $\times$ $4 \mathrm{ESM} \times 5$ locations $\times 3$ types of events) are rejected at an $\alpha=0.05$ significance level, and $8 \%$ at $\alpha=0.1$. It is concluded that the downscaled ESM trajectories in historical scenarios reproduce satisfactorily the observed distributions, so that their future counterparts can be used to project the three types of event in all the locations.

\subsubsection{Checking extrapolation in future trajectories}

An extrapolation check of the covariates is essential since, under climate change conditions, the cloud of points defined by the future covariates can be significantly shifted with respect to the observed one, used to fit the model. As in any statistical model, a frequent extrapolation may lead to unreliable projections.

Both marginal and multivariate extrapolation conditions are checked following the approach by Abaurrea et al (2015b). Briefly, given a trajectory, the intensity in day $t, \hat{\lambda}_{t}$, is obtained only if the values in that day of all the predictors are lower than their corresponding maxima in the fitting period (marginal checking). Additionally, the Mahalanobis distance of the vector of predictors in $t$ (with respect to the observed mean vector and covariance matrix) must be lower than the maximum of the Mahalanobis distances in the fitting sample or, alternatively, all the predictor values in $t$ must be lower than their $90^{t h}$ percentiles in the fitting period (multivariate checking). If the percentage of days not projected in a trajectory is greater than $25 \%$, it is removed from the analysis. 

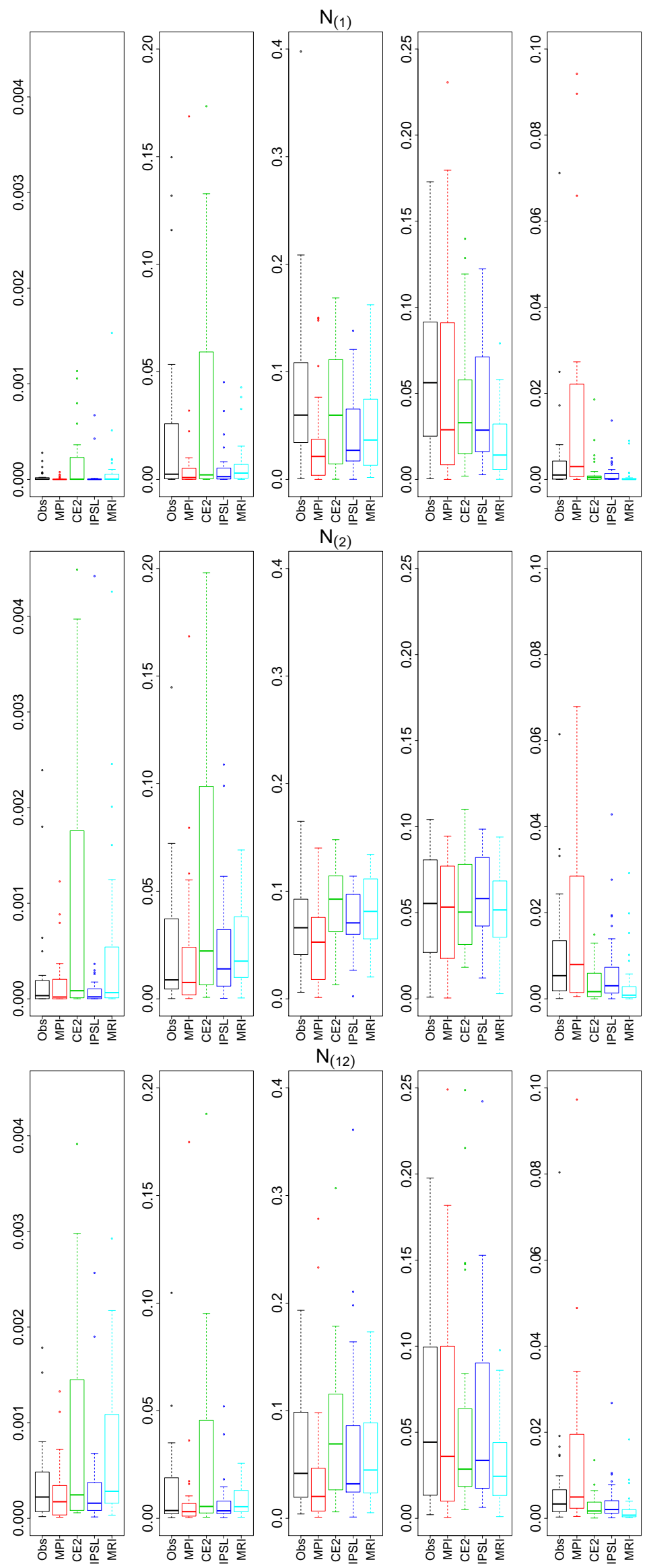

Fig. 5 Boxplots of the annual $95^{t h}$ percentiles calculated with the observed and the ESM trajectories in the historical scenario, 1971-2000, Barcelona. 


\begin{tabular}{|l|l|l|l|}
\hline \# traj. & RCP4.5 $(4$ traj) & RCP6.0 $(2$ traj) & RCP8.5 $(4$ traj $)$ \\
\hline$\geq 3$ & $86.2 \%$ & $0 \%$ & $77.3 \%$ \\
\hline 1 & $4.0 \%$ & $3.1 \%$ & $9.3 \%$ \\
\hline none & $1.8 \%$ & $3.6 \%$ & $5.3 \%$ \\
$(31-40)$ & & Alb: Jl, Au, $N_{(12)}$ & Alb: Au, $N_{(12)}$ \\
$(41-50)$ & Alb: Jl, $N_{(1)}, N_{(12)} ; \mathrm{Au}, N_{(12)}$ & & Alb: Jl, all $N_{()} ; \mathrm{Au}, N_{(1)}, N_{(12)}$ \\
$(51-60)$ & Alb: Jl, $N_{(12)}$ & Alb: Jl, Au, all $N_{()}$ & Alb: Jl, Au, all $N_{()}$ \\
\hline
\end{tabular}

Table 3 Percentage of periods (from 225) where three or more ( $\geq 3$ ), only one (1) or none of the available trajectories are projected. The location, month and indicator processes with no projection in each decade are indicated in the last three rows.

\begin{tabular}{|l|lll|lll|lll|}
\hline$\%$ & \multicolumn{3}{|c|}{ RCP4.5 (100) } & \multicolumn{3}{c|}{ RCP6.0 (50) } & \multicolumn{3}{c|}{ RCP8.5 (100) } \\
& $N_{(1)}$ & $N_{(2)}$ & $N_{(12)}$ & $N_{(1)}$ & $N_{(2)}$ & $N_{(12)}$ & $N_{(1)}$ & $N_{(2)}$ & $N_{(12)}$ \\
\hline $2031-40$ & 5 & 7 & 9 & 2 & 2 & 8 & 10 & 10 & 12 \\
\hline $2041-50$ & 10 & 12 & 13 & 2 & 0 & 2 & 16 & 19 & 15 \\
\hline $2051-60$ & 10 & 10 & 11 & 10 & 10 & 10 & 30 & 34 & 25 \\
\hline
\end{tabular}

Table 4 Percentage of non projected periods by decade. The total number of periods is in round brackets.

Extrapolation is not a big problem except in Albacete, where projections in July and August cannot be obtained. Table 3 shows the percentages from the 225 considered periods ( 5 months $\times 3$ decades $\times 3$ types of event $\times 5$ locations $)$ where three or more, only one, or none of the available trajectories are projected. Given that 2 to 4 trajectories were initially available, the results are satisfactory.

To analyse the time evolution of the extrapolation problem, Table 4 summarises the percentage of non projected periods by decade and type of event. A total of 100 periods $(5$ months $\times 4$ trajectories $\times 5$ locations $)$ are available under RCP 4.5 and RCP8.5, and $50(5 \times 2 \times 5)$ under RCP6. The maximum percentages under RCP4.5 and RCP6.0 are 13 and 10\% respectively. Under the more severe RCP8.5 the percentages increase in the third decade with a maximum value of $34 \%$ non projected periods.

\subsubsection{Summary measures to analyse the projections}

In each location, the fitted model provides the projected intensity in each day in MJJAS for the period 2031-60 (4590 days), under three RCPs and for 2 to 

385 horizontal lines. one.

4 trajectories. To deal with this huge amount of values, and since the aim is to study the general evolution of the EHE occurrence, summaries of the projected daily intensities are calculated. Robust summary measures are used to minimise the effect of the projections obtained under some extrapolation.

To study the mean evolution of the projected intensities, we use the $25 \%$ trimmed mean $\bar{\lambda}_{25}$ by month and decade, which is the mean of the daily intensities once the lowest $25 \%$ and the highest $25 \%$ values are discarded. To study the variability, the interquartile range $I Q R_{\lambda}$ is used. Since 2 to 4 trajectories are available in each location, the corresponding $\bar{\lambda}_{25}$ values of each model are summarised by their median value, $Q 2_{\bar{\lambda}_{25}}$ herein. These summary measures allow us to study the seasonal behaviour and the time evolution of the projected intensities of each type of event in each RCP, for the considered spatial area.

\subsection{Projections 2031-60 under scenario RCP4.5}

A detailed analysis of the projections obtained under RCP4.5 is shown in this section, and a comparison with the results under RCP6.0 and RCP8.5, in the next

As it was shown in Section 5.1.2 projections for Albacete could be obtained only for a few periods, and not in July and August. For that reason, the results for Albacete are not included in the figures of the following sections, although they are summarized in the tables.

Global analysis To analyse the global behaviour of the projected intensities over the area under study, the distribution of $\bar{\lambda}_{25}$ for all the trajectories in the four locations is summarised using boxplots, see Figure 6. The boxplots are displayed without the outliers to keep the y-scale short. As a reference, the minimum and maximum of the observed trimmed means in the four locations are plotted as 

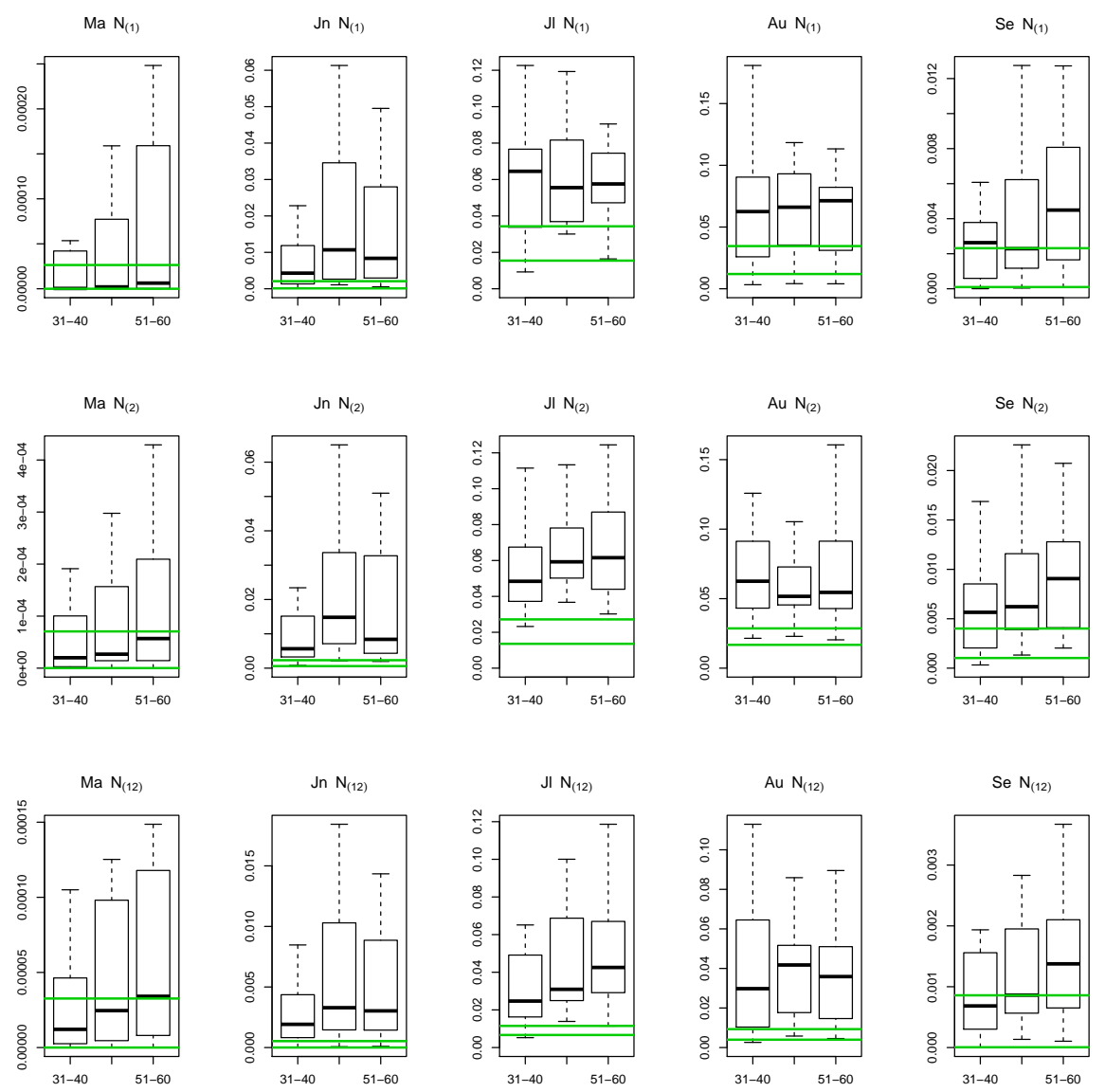

Fig. 6 Boxplots of the projected trimmed means $\bar{\lambda}_{25}$ in the four locations and all the trajectories available under RCP4.5. Green horizontal lines are the minimum and maximum of the observed $\bar{\lambda}_{25}$.

The maximum of the projected values in May is always lower than 0.0004. Since projections in this month do not lead to a relevant increase in the occurrence of EHEs and their impact is low, May will not be considered in the following analysis.

The boxplots show that the observed $\bar{\lambda}_{25}$ values from June to August are always lower than the $50^{t h}$ percentile of the corresponding projected $\bar{\lambda}_{25}$ and, in most cases, than the $25^{\text {th }}$ percentile. This fact indicates a high agreement between the different ESMs in the projection of an important increase of the three types of events. In May, June and September this variability is lower in 2031-40 than 

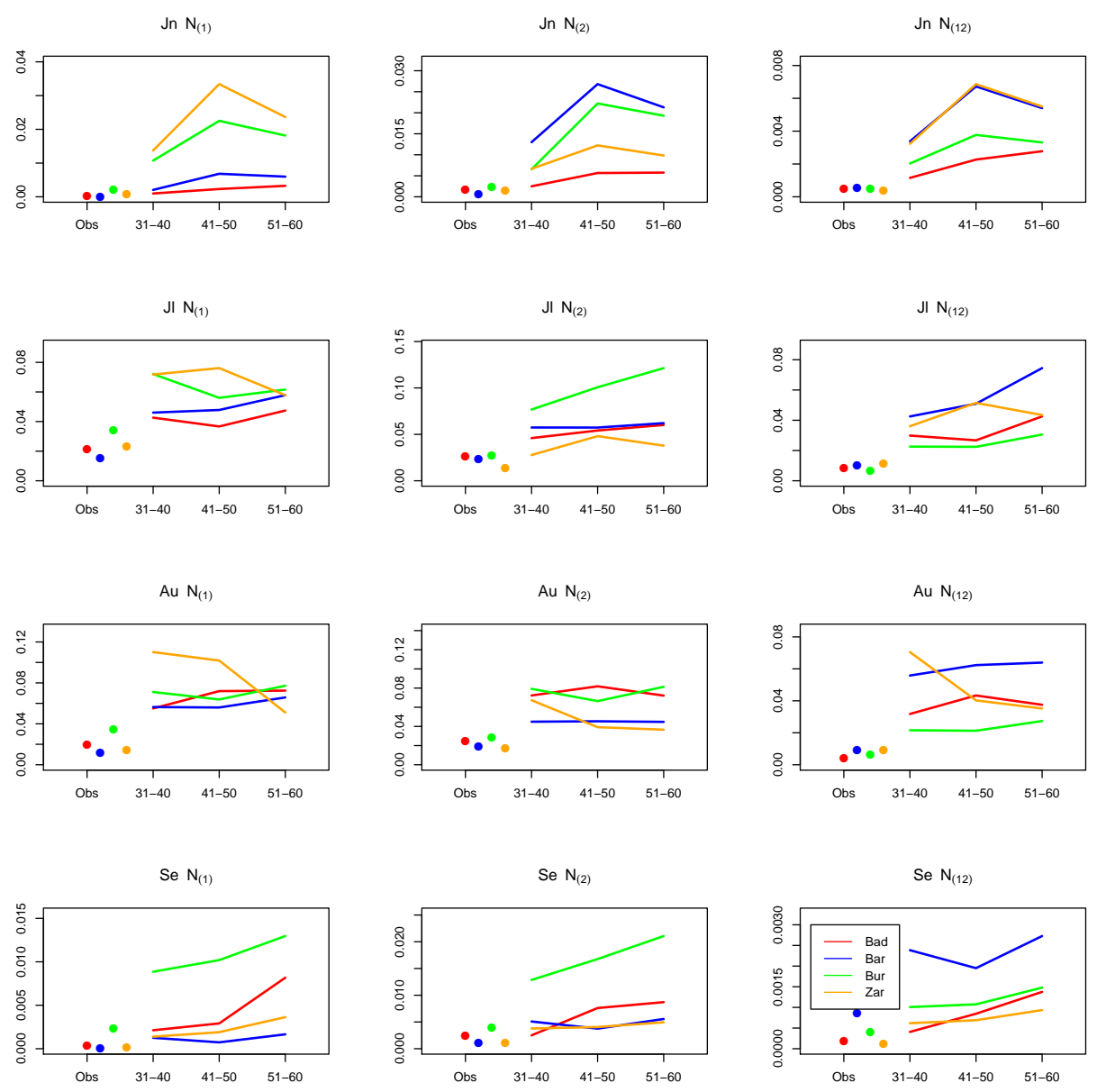

Fig. 7 Plots, by month and type of event, of $Q 2_{\bar{\lambda}_{25}}$ under RCP4.5 in the three decades and $\bar{\lambda}_{25}$ of the observed period. The projections of each location are displayed with different colours.

in the other decades. Since the variability comes from the different locations and trajectories, it means that the projections for the different locations and ESMs are more homogeneous in the first decade than later.

Time evolution To summarise and compare the time evolution of the mean level of the projections, Figure 7 shows $Q 2 \bar{\lambda}_{25}$ in the three decades and, as a reference value, the observed $\bar{\lambda}_{25}$. Most of the projected values increase from 2031-40 to 

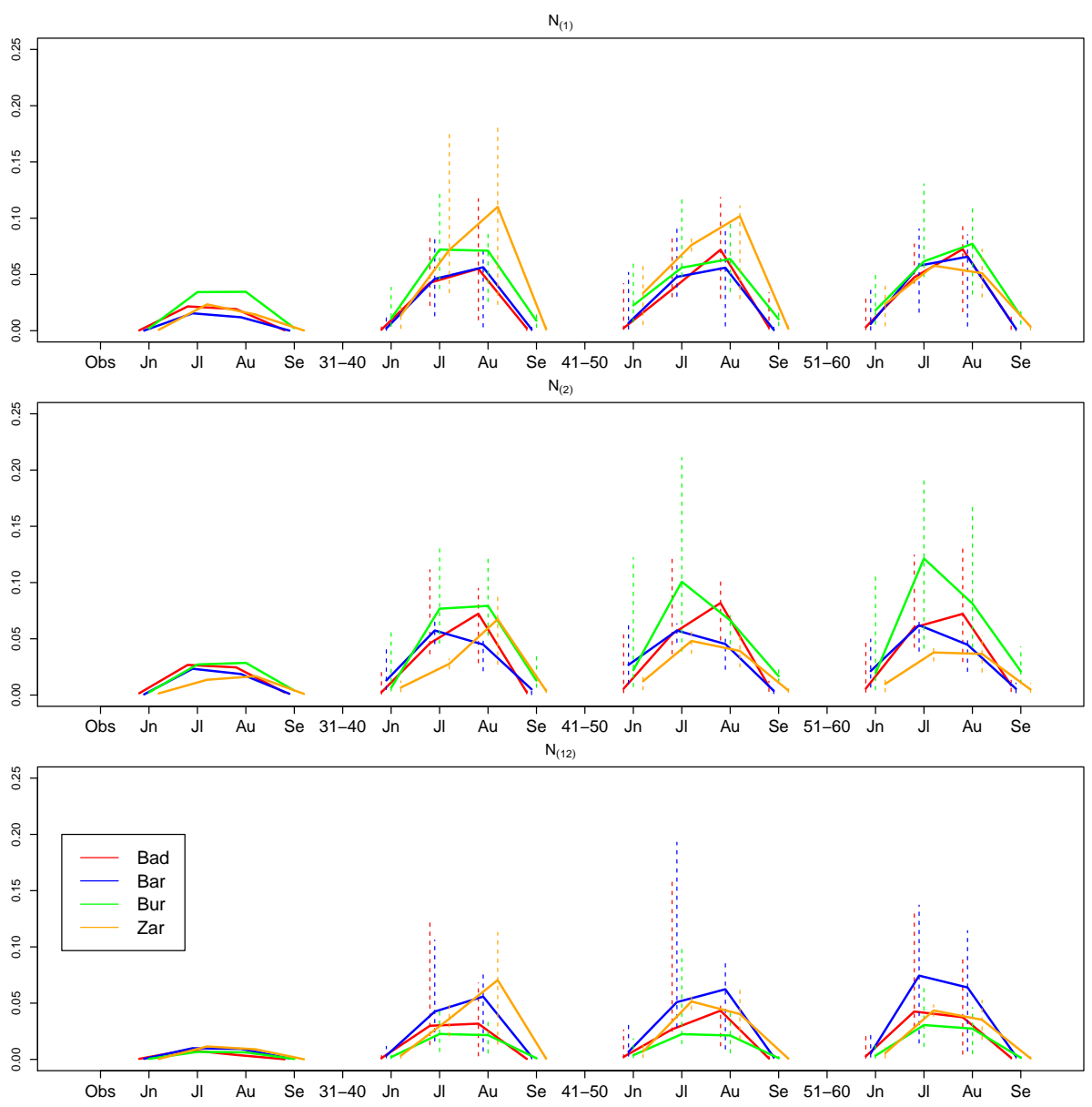

Fig. 8 Seasonal pattern of the observed $\bar{\lambda}_{25}$ and of the $Q 2_{\bar{\lambda}_{25}}$ values under RCP4.5 in 203140, 2041-50 and 2051-60. Vertical bars show the range of the $\bar{\lambda}_{25}$ values used to calculate each median.

Zaragoza, where $Q 2_{\bar{\lambda}_{25}}$ decreases in August in all type of events, and in $N_{(1)}$ also in July. The increases are more generalised in September and specially in June.

In order to analyse the time evolution of the seasonal pattern, Figure 8 displays the $Q 2_{\bar{\lambda}_{25}}$ in a different way: the monthly patterns in each decade are plotted in a row, with the observed period in the first place. Locations are displayed with different colours and the variability within the trajectories is shown by vertical bars displaying the range of the $\bar{\lambda}_{25}$ values used to calculate each median value. To make easier comparisons across the types of event and the scenarios, the same $y$-scale is used in all the plots in Figures from 8 to 11. A clear increase in the 
projected values is observed in all the months, locations and types of event, since $Q 2_{\bar{\lambda}_{25}}$ values exceed their observed counterparts in all the cases. The seasonal pattern does not show relevant differences between the three decades.

Results by type of event

$N_{(1)}$. In 2031-40, the projected increases in Barcelona and Badajoz show a similar evolution, with a median value in August greater than 0.05, while Burgos and Zaragoza show a higher increase. In August 2031-50, the $Q 2_{\bar{\lambda}_{25}}$ values in Zaragoza reach 0.1. In the last decade, the $Q 2_{\bar{\lambda}_{25}}$ values are similar in all the locations, with values from 0.047 to 0.062 in July and from 0.051 to 0.077 in August.

$N_{(2)} \cdot Q 2_{\bar{\lambda}_{25}}$ values in July and August 2031-40 move around 0.05, except in July in Zaragoza where it is 0.028 . The values in 2051-60 show more spatial heterogeneity than their counterparts in $N_{(1)}$, with the highest increase in Burgos, and the lowest one in Zaragoza.

$N_{(12)}$. As in $N_{(1)}$ and $N_{(2)}$, the levels of the projections in the three decades are quite similar. Barcelona shows the highest $Q 2_{\bar{\lambda}_{25}}$, over 0.05 , in all the months and decades, except in August 2031-40. Moreover, in 2051-60, Q2 $\bar{\lambda}_{25}$ values in $N_{(12)}$ in Barcelona are higher than their counterparts in $N_{(1)}$ and $N_{(2)} \cdot Q 2_{\bar{\lambda}_{25}}$ values in Burgos increase with respect to the observed ones, but less than in the other locations and the other types of events.

5.3 Comparison of the projections in 2031-60 under RCP4.5, RCP6.0 and RCP8.5

\subsubsection{Evolution of the mean level}

The plots of the observed $\bar{\lambda}_{25}$ and the $Q 2_{\bar{\lambda}_{25}}$ under RCP6.0 and RCP8.5 are shown in Figures 9 and 10, respectively. For easier comparison, Figure 11 summarises all the projections using different symbols for each scenario. The range of the $Q 2_{\lambda_{25}}$ corresponding to the three scenarios is displayed with dashed vertical lines. In those figures, the values of $Q 2_{\bar{\lambda}_{25}}$ which are calculated with only one trajectory 

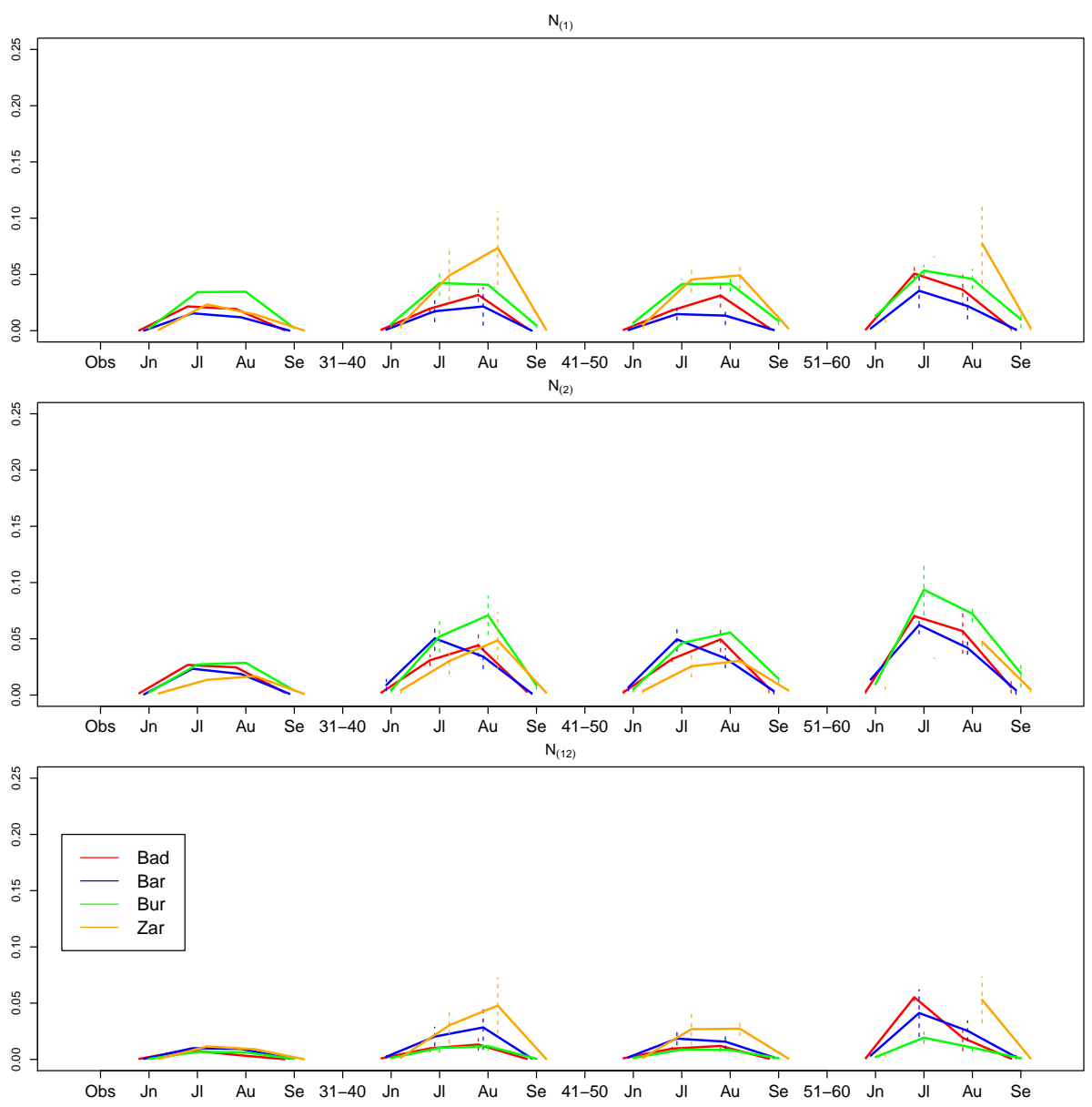

Fig. 9 Seasonal pattern of the observed $\bar{\lambda}_{25}$ and of the $Q 2_{\lambda_{25}}$ values under scenario RCP6.0, by decade. Vertical bars show the range of the $\bar{\lambda}_{25}$ values used to calculate each median.

are not plotted, since they are not real median values. The numerical values shown in these plots are also summarised in tables, see additional material: file 1 .

Scenarios. The projections under the three scenarios suggest a clear increase in the mean level of the intensity, with the $Q 2_{\bar{\lambda}_{25}}$ values under the three scenarios higher than the observed $\bar{\lambda}_{25}$. In 2031-50, the projections under RCP6.0 are smaller than under RCP4.5, as expected due to the evolution of these scenarios. However, they show a similar growth in 2051-60, except in $N_{(12)}$, where some locations show slight differences in July and August. 

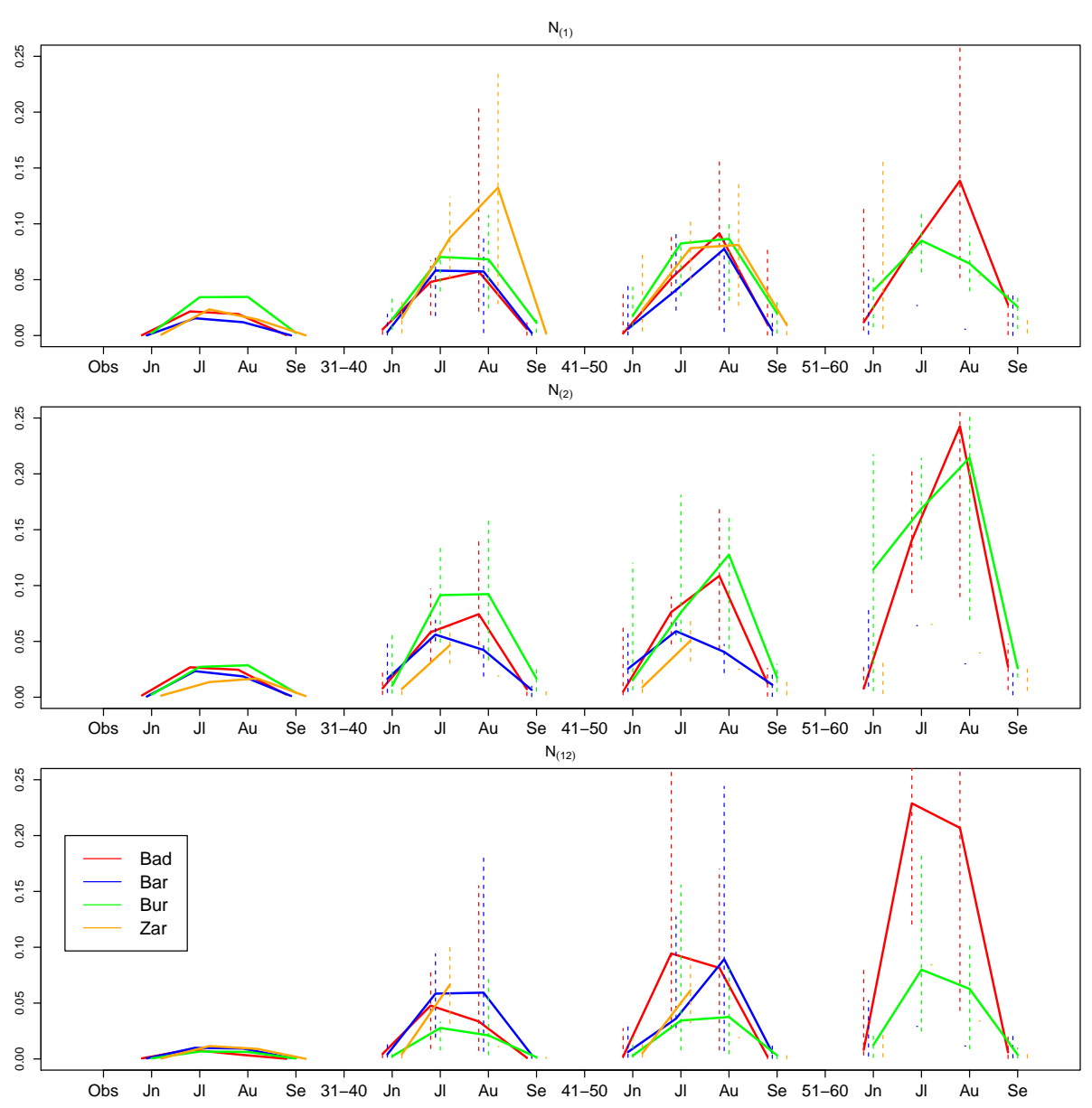

Fig. 10 Seasonal pattern of the observed $\bar{\lambda}_{25}$ and of the $Q 2 \bar{\lambda}_{25}$ values under scenario RCP8.5, by decade. Vertical bars show the range of the $\bar{\lambda}_{25}$ values used to calculate each median.

The evolution under RCP8.5 shows more relevant differences. The first is that this scenario leads to more extrapolation problems, so that less projections can be obtained. For example, in July and August 2051-60, only Badajoz and Burgos have more than one projected trajectory. In 2031-40, similar values are obtained under RCP8.5 and RCP4.5. However, in 2041-50 the projections grow faster under RCP8.5, and from 2051 onwards much higher values than in the other scenarios are projected. The wide range of the $\bar{\lambda}_{25}$ values (represented by the vertical bars) under RCP8.5 indicates that the ESMs in this RCP show a much higher variability. 

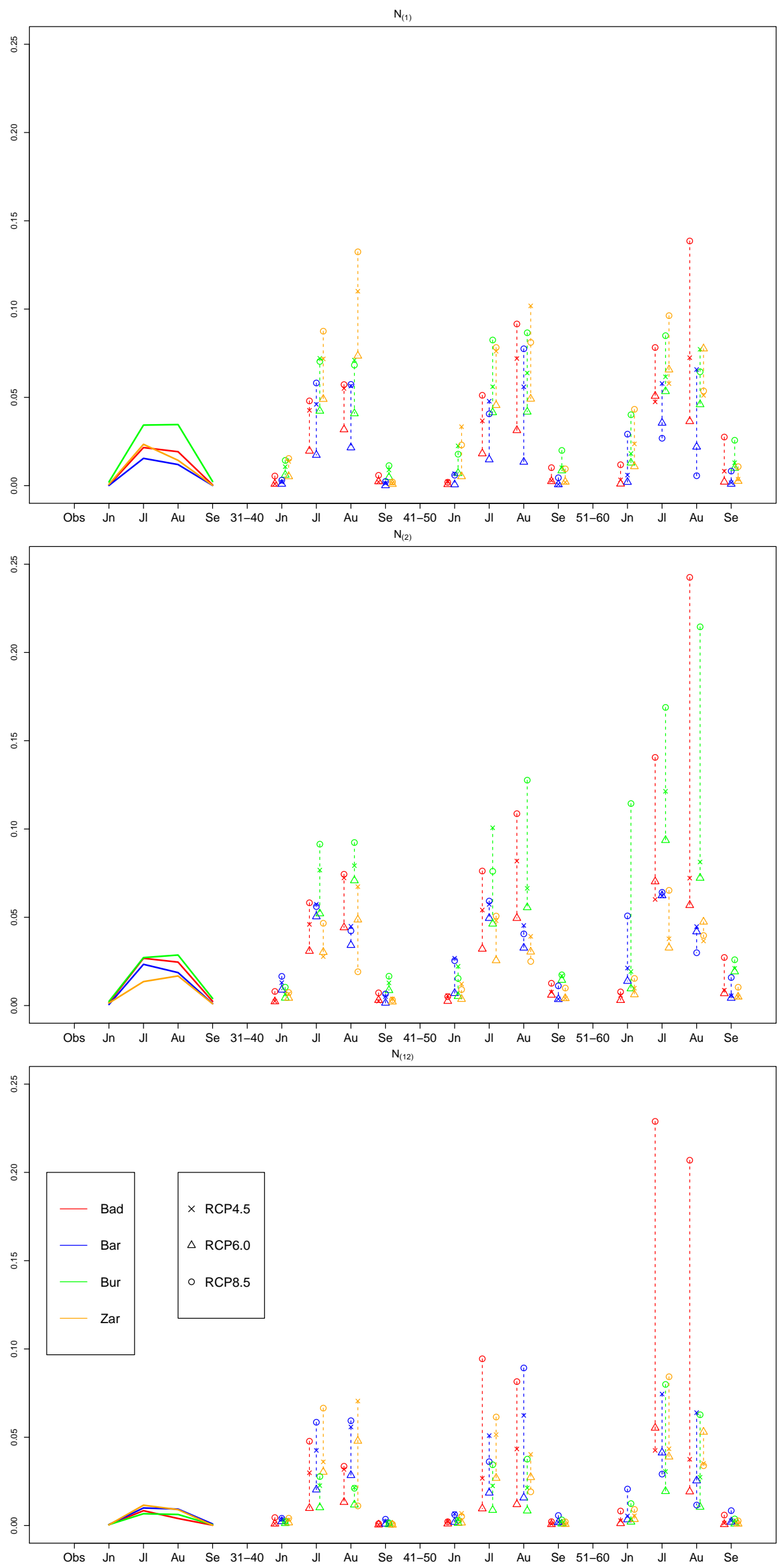

Fig. 11 Observed $\bar{\lambda}_{25}$ and $Q 2 \bar{\lambda}_{2}$ by decade and RCP. Vertical bars show the range of the projections under the different RCPs. 
Evolution by decade. In 2031-40 there are few differences between the three RCPs.

In all of them the highest intensities in $N_{(1)}$ are projected in July and August in Zaragoza (0.072 and 0.11) and in $N_{(2)}$ in Burgos, (0.077 and 0.079). In $N_{(12)}$, Burgos shows the lowest $Q 2_{\bar{\lambda}_{25}}$, around 0.02, and Zaragoza and Barcelona the highest, in all the scenarios.

The projections in 2041-50 show more variability between the scenarios. A slight increase is projected under RCP8.5, in $N_{(12)}$ and in some locations in $N_{(2)}$.

In 2051-60, the projections under RCP4.5 do not increase their mean level with respect to the previous decades, but around 16\% (10 out of 60) of the $Q 2_{\bar{\lambda}_{25}}$ values diminish. On the other hand, RCP8.5 projects a high increase in Burgos (except in $\left.N_{(1)}\right)$ and Badajoz.

Seasonal pattern. The seasonal pattern does not show important changes in any type of event, location or scenario. In all cases, the projections in June and September are higher than their observed counterparts, but they do not attain the projected values in July and August. However, in all the events and all the locations except Badajoz, the projections under RCP8.5 in June 2051-60, and sometimes even in previous decades, reach the highest observed values in July and August.

\subsubsection{Decomposition of the variability of the projections}

For a given a location, month, decade and type of event, the $\bar{\lambda}_{25}$ values corresponding to the available ESM trajectories and the three scenarios are obtained. To analyse the sources of the variability within these sets of projections, we use a sum of squares decomposition considering three factors: Location, Scenario and ESM, the latter nested in the first two. This decomposition is analogous to that performed in an ANOVA model but here it only has descriptive purposes. Similar analyses can be found in Déqué et al (2012), Räisanen and Räty (2013) and Paeth et al (2017).

Since our interest lies in the variability due to the Location and the Scenario factors, Table 5 summarises the percentages of variability explained by them, 


\begin{tabular}{|l|ll|ll|ll|}
\hline Event & \multicolumn{2}{|c|}{$N_{(1)}$} & \multicolumn{3}{c|}{$N_{(2)}$} & \multicolumn{3}{c|}{$N_{(12)}$} \\
& \% LOC & \%SCE & \%LOC & \%SCE & \% LOC & \%SCE \\
\hline Ma31-40 & & & & & & \\
May & 42.3 & 3 & 54.3 & 5.9 & 57 & 3.3 \\
June & 26.7 & 9.3 & 13.1 & 6.1 & 14.9 & 9.7 \\
July & 21.6 & 11.4 & 26.1 & 5.3 & 16.5 & 13.1 \\
August & 19 & 6.9 & 29.6 & 1.7 & 12.6 & 6.5 \\
September & 36.4 & 8.5 & 37.7 & 5.5 & 31.2 & 8.8 \\
\hline 2041-50 & & & & & & \\
May & 21.3 & 2.3 & 14 & 5 & 33 & 4.2 \\
June & 13.4 & 10.6 & 10.8 & 8.1 & 8.9 & 7.9 \\
July & 18 & 24.2 & 22.4 & 12.5 & 8.3 & 9.3 \\
August & 9.4 & 17.6 & 39.9 & 11.9 & 12 & 18.1 \\
September & 13.4 & 11 & 17.6 & 15.7 & 12.5 & 19.7 \\
\hline 2051-60 & & & & & & \\
May & 28.6 & 5.6 & 9.9 & 6.8 & 27.2 & 8 \\
June & 7.9 & 17.9 & 19.3 & 11.3 & 7.4 & 15.8 \\
July & 16.3 & 9.3 & 34.1 & 20 & 18 & 23.9 \\
August & 17.3 & 9.3 & 27.7 & 24.4 & 14.6 & 15.5 \\
September & 16.4 & 23.7 & 16.3 & 18.3 & 9.7 & 23.4 \\
\hline
\end{tabular}

Table 5 Percentage of variability within the sets of projections explained by the factors Location (\%LOC) and Scenario (\%SCE).

\% LOC and \%SCE respectively. A low percentage \%LOC (\%SCE) indicates that the differences between the locations (scenarios) are less relevant than the other sources of variability. Differences between scenarios grow over time, with the median of \%SCE equal to $6.9 \%$ in $2031-40$ and to $15.8 \%$ in $2051-60$. The main conclusions are summarised below by type of event.

$N_{(1)}$. In the first decade, the projections show differences between locations but they are similar under the three scenarios, with \% SCE percentages lower than $12 \%$. The projections in all the locations are more similar from 2041, with \%LOC values lower than $20 \%$ except in May.

$N_{(2)}$. The variability between locations is higher in this type of events, with \% LOC values greater than $22 \%$ in July and August in the three decades and only 4 (out of 15 ) lower than 16\%. The variability between scenarios is low, with 12 out of 15 of the \% SCE values lower than 16\%. In July and August, the sum of the variability of both factors increases gradually from the first to the third decade, which is consistent with the values in Figure 11.

$N_{(12)}$. The variability between locations is in general low, with all the values lower than $19 \%$ except those in May and one in September. The variability between 

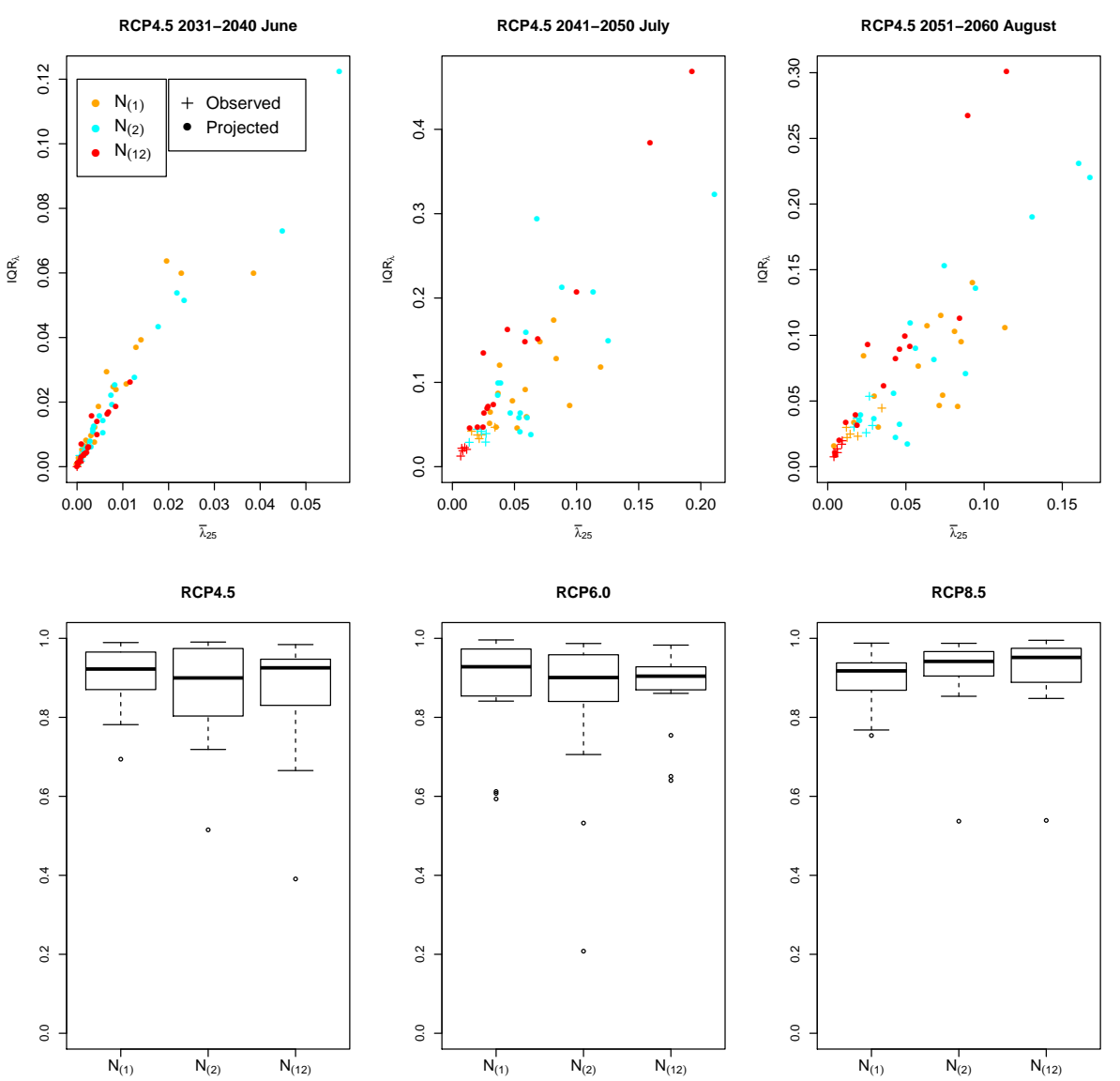

Fig. 12 Plots of $I Q R_{\lambda}$ versus $\bar{\lambda}_{25}$ for a month in each decade under RCP4.5 (top row) and boxplots of the correlation coefficients between $I Q R_{\lambda}$ and $\bar{\lambda}_{25}$ under the three RCPs (bottom row)

scenarios is also low, with all the \% SCE values lower than $20 \%$ except in the last

decade, which shows a greater variability.

\subsubsection{Evolution of the variability of the projected daily intensities}

In this section, the evolution of the variability of the projected daily intensities is studied using the interquartile range $I Q R_{\lambda}$ defined in Section 5.1.3.

First, the relationship between the mean level and the variability of the intensities is checked graphically. A strong linear positive relation is found in most 
cases, see as an example the top row in Figure 12, where the plots of $I Q R_{\lambda}$ versus $\bar{\lambda}_{25}$, for the three types of events are shown in June 2031-40, July 2041-50 and August 2051-60 under RCP4.5.

This linear relationship is quantified using the correlation coefficient. Given the high number of coefficients (around $540=5$ months $\times 3$ decades $\times 3$ RCPs $\times$ 3 types of event $\times 4$ locations), they are summarised using boxplots by type of event and scenario, see bottom row in Figure 12. The median of the coefficients under RCP4.5, RCP6.0 and RCP8.5 are 0.92, 0.91 and 0.93 respectively, and the first quantiles, $0.84,0.86$ and 0.88 . In all the scenarios, more than $82 \%$ of the coefficients are greater than 0.8 . This high correlation between the mean and the variability suggests that the conclusions of the projected change for the mean level are also valid for the variability.

A sum of squares decomposition of the variability of the sets of $I Q R_{\lambda}$ values (not shown) leads to similar conclusions to those obtained for $\bar{\lambda}_{25}$ in Section 5.3.2. The variability explained by the scenarios is low, lower than $16 \%$ in $2031-40$. The variability between locations is higher than between scenarios, except in 9 cases out of 45 . In general, $N_{(2)}$ shows the highest \%LOC values (most of them higher than $23 \%$ ), and $N_{(12)}$ the lowest.

All these results show that the dispersion of the projected daily intensities will be greater than that of the observed intensities, in all the decades and scenarios. Hence, the increase in the mean frequency of EHEs will be accompanied by an increase in the variability of that frequency, so that a very high number of EHEs can be expected in some years during the next decades.

\subsection{Comparison with other works}

Projections of high percentiles of $T x_{t}$ in summer have been obtained to analyse the future changes in the upper tail of temperature distributions, see for example El Kenawy et al (2015) for a study in the Ebro Valley (NE Spain). However, as far as we know, there are no projections of the occurrence of EHEs in Spain. This 
section summarises the conclusions drawn in other studies about projections of the occurrence of heat waves in nearby areas, for time periods around mid $21^{\text {st }}$ century. It must be taken into account that they are based on different heat wave definitions, so that a direct comparison is not possible. However, our results are generally consistent with them.

Lemonsu et al (2014) carried out a study with a similar objective, the analysis of the temporal evolution of heat wave frequency in the Paris area under A1B, A2 and B1 scenarios for 2020-49 and 2070-99. Their heat wave definition is based on the moving average of daily maximum and minimum temperatures over 3 days and it is applied to RCM projections. They found a systematic increase in the mean number of heat waves: 1 every 7 years during the observed period, 1 every 2 years in 2020-49, and between 1 and 2 every year in 2070-99. This means that the projected increase ratio between 2020-49 and the observed period is around 3.5. In our case, the median of the projected increase ratios between 2031-40 and 1971-2000 in July and August is 3.5 for the simultaneous events, 2.2 for $N_{(1)}$ and 2.4 for $N_{(2)}$.

Pereira et al (2017) analysed the occurrence of heat waves, defined only with $T x$, in 12 locations in the Iberian Peninsula. They compared the observed values in 1986-2005 with those projected in 2046-2065 using a RCM forced with MPI-ESMLR under RCP8.5. They found statistically significant changes in the frequency of occurrence in Barcelona, with a projected/observed ratio of 7.9. Some other locations next to those considered in this paper are also analysed: Cáceres with a ratio of 3.4, Madrid with 3.8 and Sevilla with 3.1. These results are consistent with our projections in 2041-50 for $N_{(1)}$ under RCP8.5, where the ratios in July and August are 2.6 and 6.5 in Barcelona, 2.4 and 4.8 in Badajoz, and 3.3 and 5.7 in Zaragoza.

Fischer and Schär (2010) analysed future changes in summer heat waves using six RCMs of the ENSEMBLES multi-model experiment with simulations forced with the SRES A1B scenario. They found that in the Iberian Peninsula and the 
Mediterranean region, the frequency of heat waves per summer will increase from an average of about 0.2 in 1961-90 to around 1.3 in 2021-50, so that the increasing factor is around 6.5. They also studied the frequency of days with $T x>35^{\circ} \mathrm{C}$ and $T n>20^{\circ} \mathrm{C}$, which is a similar concept to that of simultaneous events. The increasing factor of this frequency between the same periods is 2.3 .

Lau and Nath (2014) obtained projections of the occurrence and intensity of spatial heat waves in western Europe, including France and Germany but not the Iberian Peninsula, under RCP4.5 and using the GFDL high resolution atmospheric model (HiRAM) with 50-km grid spacing. They found that the frequency of heat waves projected in 2026-35 will increase by a factor 3.3 with respect to the frequency observed in 1979-2008.

\section{Conclusions}

In this work, we propose a statistical model for extreme heat events which can be used to obtain future projections of the occurrence of those events at a daily and local scale. It is shown that the suggested approach is useful to obtain projections at those scales, where the dynamic climate models show difficulties, and which are required in climate change impact studies and other applications.

Occurrence model of extreme heat events. A non homogeneous common Poisson shock process is applied to jointly model the occurrence of extreme heat events in maximum and minimum daily temperature series in five Spanish locations. The NHCPSP is made up of three conditionally independent Poisson processes which model the occurrence of EHEs only in $T x_{t}$, only in $T n_{t}$ and in both temperatures simultaneously.

The set of potential covariates in the models includes harmonic terms, short term temperature moving means, $T x_{m 15}, T n_{m 15}, T x_{m 31}$ and $T n_{m 31}$, polynomial functions of them and interactions with the harmonic terms. The final fitted models 
are simple, including only one harmonic and linear temperature terms in most cases. All of them are satisfactorily validated.

Projection methodology. The fitted models are useful for obtaining local projections of the intensity of the EHE occurrence under climate change conditions. These conditions are described by the covariates obtained from the future temperature trajectories generated by ESMs, appropriately downscaled to fit the local characteristics. Trajectories from RCMs could also be used.

In order to obtain reliable projections, two issues have to be checked. First, that the considered trajectories reproduce adequately the current climate and second, that the models are not used under severe marginal or multivariate extrapolation conditions. Simple tools to check these requirements are provided. This approach has proved to be generally useful for medium-term projections, since four out of the five locations considered passed the extrapolation control.

To analyse the projected daily intensities, two summary measures, the $25 \%$ trimmed mean $\bar{\lambda}_{25}$ for the mean level, and the interquartile range $I Q R_{\lambda}$ for the variability are suggested.

Results of the EHE projections. The most relevant feature of the projections in 2031-60 is the high increase in the intensities, specially in July and August. The projections in June and September are higher than their observed counterparts in all the cases, but they do not attain the projected values in July and August. However, the projections under RCP8.5 in June in the last decade reach the observed values in July and August, except in Badajoz.

Projections under RCP4.5 and RCP8.5 are quite similar in 2031-40, but in the following decades a high increase is projected under RCP8.5, while there is no increase under RCP4.5 nor RCP6.0.

It is noteworthy the high increase projected in the occurrence of simultaneous events $N_{(12)}$. Although this type of events shows the lowest intensities in the observed period, it shows the highest ratio projected/observed intensities in 2031- 
40. More precisely, under RCP4.5 and RCP8.5, the frequency in $N_{(12)}$ in July and August from 2031 onwards will be more than three times higher than in the observed period.

Concerning spatial behaviour, RCP6.0 shows the lowest variability of the three scenarios and RCP8.5 the highest. It is also observed that different evolutions are projected in locations with the same Köppen climate classification, such as Badajoz and Barcelona. There is not any spatial pattern, except in $N_{(2)}$, where Burgos shows the highest projected intensities in all the scenarios and decades.

The conclusions about the projected change for the mean level of the occurrence intensities are also valid for its variability. This result is determined by the high correlation found between the mean level and the variability summary measures, $\bar{\lambda}_{25}$ and $I Q R_{\lambda}$.

Future work. The suggested approach is not useful for obtaining long-term projections of the EHE occurrence due to the extrapolation problem, and even over a medium time horizon it may not be adequate in some cases. We intend to use this type of model with other atmospheric covariates to obtain projections up to 2100. These covariates also reflect the climate change conditions, but they have a lower explicative capacity of the EHE process. Their advantage is that they do not lead to severe extrapolation, unlike the temperature variables.

Acknowledgements The authors acknowledge the financial support from the Ministerio de Ciencia e Innovación (Spanish Department of Science) and the Ministerio de Medio Ambiente (Spanish Department of Environment) through projects CGL2009-09646 and ESTCENA 2009/0017. They thank the anonymous reviewers for their helpful comments and AEMET, the Spanish meteorological agency, and specially $\mathrm{M}^{a}$ Jesús Casado for supplying the temperature data.

\section{References}

Abaurrea J, Asín J, Cebrián AC, Centelles A (2007) Modeling and forecasting extreme heat events in the central Ebro valley, a Continental-Mediterranean 
area. Global Planet Change 57(1-2):43-58

Abaurrea J, Asín J, Cebrián AC (2015a) A bootstrap test of independence between three temporal nonhomogeneous Poisson processes and its application to heat wave modeling. Environ Ecol Stat 22(1):127-144

Abaurrea J, Asín J, Cebrián AC (2015b) Modeling and projecting the occurrence of bivariate extreme heat events using a non-homogeneous common Poisson shock process. Stoch Environ Res Risk Assess 29:309-322

AEMET (2011) Atlas climático ibérico/Iberian climate atlas. Agencia Estatal de Meteorología, Ministerio de Medio Ambiente y Rural y Marino, Madrid, Instituto de Meteorologia de Portugal

Amengual A, Homar V, Romero R, Brooks H, Ramis C, Gordaliza M, Alonso S (2014) Projections of heat waves with high impact on human health in Europe. Global Planet Change 119(0):71 - 84

Barriopedro D, Fischer EM, Luterbacher J, Trigo RM, García-Herrera R (2011) The hot summer of 2010: Redrawing the temperature record map of Europe. Science 332(6026):220-224

Beniston M, Stephenson D, Christensen O, Ferro C, Frei C, Goyette S, Halsnaes K, Holt T, Jylhä K, Koffi B, Palutikof J, Schöll R, Semmler T, Woth K (2007) Future extreme events in European climate: an exploration of regional climate model projections. Climatic Change 81(1):71-95

Brands S, Herrera S, Fernández J, Gutiérrez JM (2013) How well do CMIP5 Earth System Models simulate present climate conditions in Europe and Africa? Clim Dyn 41:803-17

Casanueva A, Herrera S, Fernández J, Frías MD, Gutiérrez JM (2013) Evaluation and projection of daily temperature percentiles from statistical and dynamical downscaling methods. Nat Hazard Earth Sys 13:2080-99

Cattiaux J, Douville H, Peings Y (2013) European temperatures in CMIP5: origins of present-day biases and future uncertainties. Clim Dyn 41(11):2889-2907 
Cebrián AC, Abaurrea J, Asín J (2015) NHPoisson: An R package for fitting and validating nonhomogeneous poisson processes. J Stat Softw 64(6):1-25

Cheng L, Agha Kouchak A, Gilleland E, Katz RW (2014) Non-stationary extreme value analysis in a changing climate. Climatic Change 127(2):353-69

Coles S (2001) An Introduction to Statistical Modeling of Extreme Values. Springer-Verlag

Déqué M, Somot S, Sánchez-Gómez E, Goodess CM, Jacob D, Lenderink G, Christensen OB (2012) The spread amongst ENSEMBLES regional scenarios: regional climate models, driving general circulation models and interannual variability. Clim Dyn 38:951-64

El Kenawy A, López-Moreno JI, McCabe MF, Brunsell NA, Vicente-Serrano SM (2015) Daily temperature changes and variability in ENSEMBLES regional models predictions: Evaluation and intercomparison for the Ebro Valley (NE Iberia). Atmos Res 155:141-57

Fischer EM, Schär C (2010) Consistent geographical patterns of changes in highimpact European heatwaves. Nature Geoscience 3:398-403

Furrer EM, Katz RW, Walter MD, Furrer R (2010) Statistical modeling of hot spells and heat waves. Clim Res 43(3):191-205

García-Cueto OR, Cavazos MT, de Grau P, Santillán-Soto N (2014) Analysis and modeling of extreme temperatures in several cities in Northwestern Mexico under climate change conditions. Theor and Appl Climatol 116(1-2):211-25

Grotjahn R, Black R, Leung R, Wehner MF, Barlow M, Bosilovich M, Gershunov A, Gutowski WJ, Gyakum JR, Katz RW, Lee YY, Lim YK, Prabhat (2016) North American extreme temperature events and related large scale meteorological patterns: a review of statistical methods, dynamics, modeling, and trends. Clim Dyn 46(3):1151-1184

Gutiérrez J, San-Martín D, Brands S, Manzanas R, Herrera S (2013) Reassessing statistical downscaling techniques for their robust application under climate change conditions. J Clim 26:171-88 
Hajat S, Armstrong B, Baccini M, Biggeri A, Bisanti L, Russo A, Paldy A, Menne B, Kosatsky T (2006) Impact of high temperatures on mortality: is there an added heat wave effect? Epidemiology 17(6):632-8

Keellings D, Waylen P (2014) Increased risk of heat waves in Florida: Characterizing changes in bivariate heat wave risk using extreme value analysis. Applied Geography 46:90 - 97

Keellings D, Waylen P (2015) Investigating teleconnection drivers of bivariate heat waves in Florida using extreme value analysis. Clim Dyn 44(11):3383-3391

Kyselý J, Picek J, Beranová R (2010) Estimating extremes in climate change simulations using the peaks-over-threshold method with a non-stationary threshold. Global Planet Change 72(1):55-68

Lau N, Nath M (2014) Model simulation and projection of European heat waves in present-day and future climates. J Clim 27:3713-30

Lemonsu A, Beaulant AL, Somot S, Masson V (2014) Evolution of heat wave occurrence over the Paris basin (France) in the 21st century. Clim Res 61:75-91

Meehl G, Washington WM, Collins W, Arblaster J, Hu A, Buja L, Strand W, Teng H (2005) How much more global warming and sea level rise? Science 307:1769-72

Paeth H, Vogt G, Paxian A, Hertig E, Seubert S, Jacobeit J (2017) Quantifying the evidence of climate change in the light of uncertainty exemplified by the Mediterranean hot spot region. Global Planet Change 151:144-151

Pereira SC, Marta-Almeida M, Carvalho AC, Rocha A (2017) Heat wave and cold spell changes in Iberia for a future climate scenario. International Journal of Climatology URL http://dx.doi.org/10.1002/joc.5158

Perkins SE, Alexander LV (2013) On the Measurement of Heat Waves. J Clim 26(13):4500-4517

Räisanen J, Räty O (2013) Projections of daily mean temperature variability in the future: cross-validation tests with ENSEMBLES regional climate simulations. Clim Dyn 41:1553-68 
Rosenbaum PR (2005) An exact distribution-free test comparing two multivariate distributions based on adjacency. J R Stat Soc B 67:515-30

Sánchez de Cos C, Sánchez J, Jiménez C, Rodríguez E (2016) Using feedback from summer subtropical highs to evaluate climate models. Atmc Sci Let 17:230-235

Smith T, Zaitchik B, Gohlke J (2013) Heat waves in the United States: definitions, patterns and trends. Climatic Change 118(3):811-825

Tobías A, Armstrong B, Gasparrini A, Diaz J (2014) Effects of high summer temperatures on mortality in 50 Spanish cities. Environmental Health 13(1):48

Tryhorn L, Risbey J (2006) On the distribution of heatwaves over the Australian region. Aust Meteorol Mag 55:169-82

Vautard R, Gobiet A, Jacob D, Belda M, Colette A, Déqué M, Fernández J, GarcíaDíez M, Goergen K, Güttler I, Halenka T, Karacostas T, Katragkou E, Keuler K, Kotlarski S, Mayer S, van Meijgaard E, Nikulin G, Patarčić M, Scinocca J, Sobolowski S, Suklitsch M, Teichmann C, Warrach-Sagi K, Wulfmeyer V, Yiou P (2013) The simulation of European heat waves from an ensemble of regional climate models within the EURO-CORDEX project. Clim Dyn 41(9-10):25552575

Wang X, Yang T, Shao Q, Acharya K, Wang W, Yu Z (2012) Statistical downscaling of extremes of precipitation and temperature and construction of their future scenarios in an elevated and cold zone. Stoch Environ Res Risk Assess 26(3):405-18

Wilby R, Dawson C (2013) The statistical downscaling model: insights from one decade of application. International Journal of Climatology 33(7):1707-19

Yue T, Zhao N, Fan Z, Li J, Chen C, Lu Y, Wang C, Xu B, Wilson J (2016) CMIP5 downscaling and its uncertainty in China. Global Planet Change 146:30-37 